\author{
Available online at https://www.rjtl.org \\ Volume: 2, Issue: 2, 85-87, 2021 \\ ISSN: $2708-3632$ \\ DOI: https://doi.org/10.46590/rjtl.2021.020203
}

\title{
Development of Non-Woven Fabrics from Bamboo/Polypropylene Fibres and its Application in Personal Protective Equipment-A Review.
}

\author{
Kanchana Devi ${ }^{1}$, Rajkumar $\mathbf{G}^{2}$ \\ ${ }^{1}$ M.Tech Scholars, ${ }^{2}$ Assistant Professor, \\ Dept of Fashion Technology, Kumaraguru College of Technology, Coimbatore- 641049, India.
}

Paper History

Received: March 2021

Accepted: April 2021

Published: May 2021

Corresponding Author

Kanchana Devi

kanchanadevi.19mat@kct.ac.in

\begin{abstract}
The important component of personal protective equipment is a mask. Mask is more important in a pandemic because it safeguards our life by stopping the spreading of the Coronavirus by entrapping the droplets from the corona affected person to enter into the wearer's nose. Many types of masks are available such as non-woven mask, reusable mask, N95 mask and cloth mask etc. The majority of commercial masks are non-woven masks which are made up of polypropylene fibre. The main drawback of non-woven masks is their discomfort due to lack of breathability. This problem is solved by utilizing natural fibres as one of the raw material in non-woven and using them in the mask. Bamboo fibre is a natural celluloid fibre having good comfort properties with antimicrobial properties. Hence an attempt is made in this review article to examine the important properties of bamboo fibre and to evaluate its potential as a protective barrier material in non-woven face masks.
\end{abstract}

Keywords: Bamboo Fibre, Breath Ability, Comfort, Face Mask, , Polypropylene Fibre.

Citation: Kanchana Devi, Rajkumar G.," Development Of Non-Woven Fabrics From Bamboo/Polypropylene Fibres And Its Application In Personal Protective Equipment-A Review..” Research Journal of Textile and Leather, 2(2), 85-87, 2021.

\section{INTRODUCTION}

Globally, there is a huge demand for Personal Protective Equipment (PPE) in Covid situation. PPE protects the user against health/safety risks at work. PPE include items such as safety helmets, gloves, mask, high-visibility clothing and so on. Normally raw material used for PPE are polypropylene fibres. The polypropylene fibres are synthetic fibre which are non-biodegradable. So PPE developed from polypropylene fibres has different drawback like lack of breath ability and odor/smell. [1].

Bamboo has the fastest growth rate among the various types of renewable natural fibres. The cellulose fibres in bamboo are aligned along the length of the bamboo providing maximum tensile strength, flexural strength, and rigidity in that direction even though bamboo is abundantly available in most of the tropical countries it has not been exploited to its potential as a reinforcing agent. So that to improve the comfort properties and reduce the polypropylene content, the addition of natural fibre like bamboo can be used.
Hence a review has been made in this paper about bamboo and polypropylene fibres in development of face mask. Also the various mechanical, chemical, and biological approaches for the preparation and separation of bamboo fibre reinforced with polypropylene has also been discussed.

\section{PREPARATION OF BAMBOO FIBRE}

Bamboo fibre have two preparation methods

- Mechanical method

- Chemical method

Mechanical process of bamboo fibre and contain flax production, while chemical process are of two varieties namely bamboo viscose rayon and bamboo lyocell.

\subsection{Properties Of Bamboo Durability}

To improve the durability of bamboo fibre and to provide high strength, the bamboo fibre is reinforced with polypropylene. Bamboo fibre strengthen polypropylene composite has high fatigue resistance results in its extreme durability [2]. 


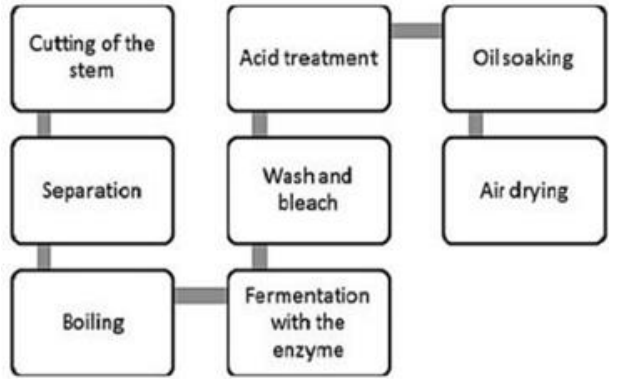

Fig.1.Bamboo Fibre Preparation Procedure

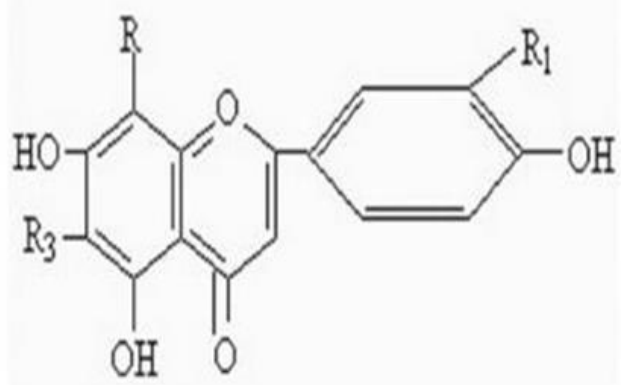

Fig.2. Structure of Bamboo Fibre

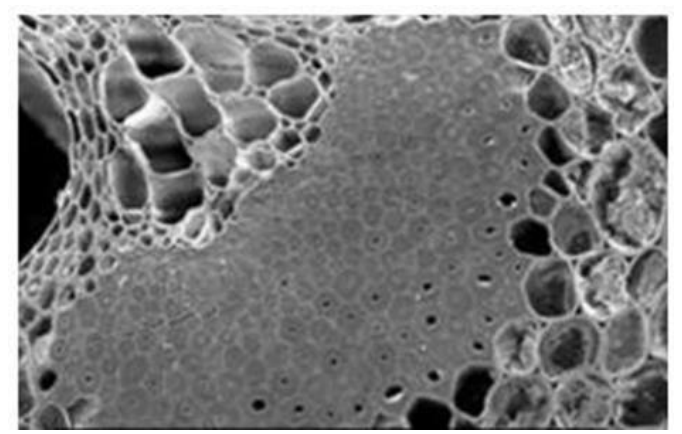

Fig.3. Cross Sectional View of Bamboo Fibre.

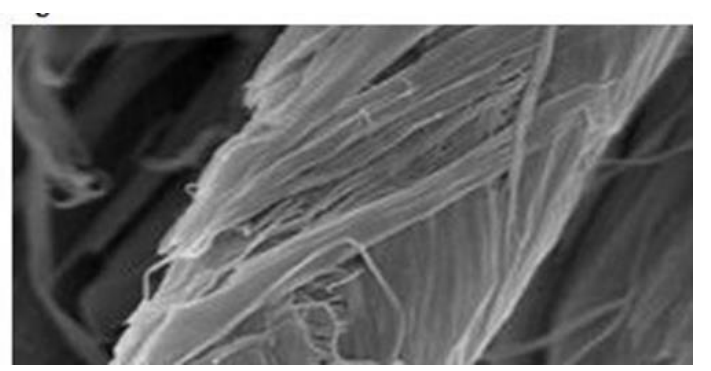

Fig.4.Longitudinal View

\subsection{Elasticity}

When external layer of bamboo is expelled, specific gravity is brought down and thus elasticity decreased. When bamboo fibre steam is exploded into poly lactic acid matrix, it is observed to have high elasticity. There is decrease in modules of flexibility between longitudinal and transverse heading, the modulus of flexibility of longitudinal heading is 16.1pa [3].

\subsection{Elongation}

Bamboo when treated with caustic pop of distinctive concentration appear significant decrease in percent prolongation at break with increment in concentration of caustic soda[4].

\subsection{Hardness}

The hardness of bamboo decrease due to steaming treatment.Tangle micro-fibrillate cellulose fibre when include to poly lactic acid/bamboo fibre composite, increment the hardness and anticipate break improvement, bamboo $23 \%$ harder than oak and 13\% harder than shake maple[5].

\subsection{Impact Strength}

Affect quality of bamboo fibre concrete sis unmistakably higher [6]. The affect the quality of poly lactic acid/bamboo fibre composite increment after expansion of micro fibrillate cellulose[4].

\subsection{Application Of Bamboo Fibre}

Bamboo texture are made by unadulterated bamboo fibre yarn,which have great damp penetrability, dampness vapour transmission property ,delicate hand,way better drapery, simple passing on impressive colour .It could be a recently established,extraordinary imminent green texture[7].

\subsection{Bamboo Hint Apparel}

It includes sweater, bath-suit, mat, cover, towel have comfortable hand, extraordinary gloss and shinning colour, great water absorbance. Bamboo fibre has such a sole work as against microscopic organism which is appropriate to create clothing tight t-shirt and socks. Its anti-ultraviolet nature is appropriate to create summer clothing particularly for the assurance of pregnant women and children from the harmed of bright radiation[7].

\subsection{Bamboo Sterile Material}

In this material have gauze, cover, surgical dress, medical caretaker wear and so on. The bamboo fibre has normal impact of sterilization and bacteriology, in this manner it has especially wide closer view on application in sterile fabric as sterile tower, cloth cover, permeable cushion, nourishment pressing and so on[7].

\subsection{Construction Material}

Bamboo is one of the most seasoned and most flexible building material. Bamboo has certain qualities due to which it can be utilized for development purpose. These qualities incorporate its hardness and lightweight .Bamboo does not 
required preparing or finishing .Bamboo development are solid and safe to indeed soil tremors[8].

\subsection{Pharmaceutical Industry}

Eating bamboo fibre diminishes the rate of intestinal characteristic flora and pathogen. This property is connected to create a bamboo medicate for gastrointestinal contamination [9].

\subsection{Textile Industry}

Normal bamboo fibres have a few of the fabulous properties, which make it a very potent fabric to be utilized in material industry. Refined bamboo fibres with moo non- celluloid substance can be utilized in materials [10].

\subsection{Determination of Bamboo/Polypropylene}

The advancement within the field of material innovation with innovations in normal and artificial material,that point to enhance the consolation properties and cleanliness of the Clint in medical field.The fibre utilized within the therapeutic PPE should be non-toxicity.non allergic, capacity to sterilized and possess mechanical properties such as quality and make them accept they are amazingly safer.Polypropylene spun bond,spun bond melt-blow ,spun bind texture are utilized in fabrication of non woven PPE[11].

\subsection{Merits of Bamboo Fibre}

Three layers of $100 \%$ bamboo woven fabric with a thread count of 800 . This fabric, used for our bedsheets, is tightly-woven, therefore hindering particles from permeating the mask. Bamboo fibre, being naturally antibacterial, inhibits bacterial growth. Silky and soft, they don't irritate the skin. They are $50 \%$ cooler than cotton, so a great choice for the hot summer. Breathable, which is important. If the mask is not breathable, the user tends to suck in and inhale air from the sides, which not filtered defeats the purpose of the mask. Our bamboo masks don't leave any compression marks on your face.

\section{CONCLUSION}

It is concluded from this review paper that bamboo fibre has high potential ability in non-woven manufacturing and utilize them as a raw material in face mask. The bamboo fibre are been used in many application of medical textile due to its excellent natural anti-microbial property. The fibre also act as a good thermal insulating material and it is biodegradable. This type of natural fibre when reinforced with polypropylene, the strength and durability of the fabric can be increased and also possess good barrier property and good breath ability which is required for a face mask. Thus in this paper i conclude when a natural fibre blend face mask provide high breath ability with good barrier properties. In bamboo fibre have good breath ability so that we prefer a face mask in natural fibre.

\section{REFERENCES}

[1]. Liu, D., Song, J., Anderson, D. P., Chang, P. R., \& Hua, Y. (2012). Bamboo fibre and its reinforced composites: structure and properties. Cellulose, 19(5), 1449-1480.

[2]. Thwe MM, Liao K (2002a) Effects of environmental aging on mechanical properties of bamboo-glass fibre reinforced polymer matrix hybrid composites. Compos Part A Appl Sci Manuf 33(1):4352.

[3]. Yu Y, Fei B, Zhang B, Yu X (2007) Cellwall mechanical properties of bamboo investigated by in-situ imaging nanoindentation. Wood Fibre Sci 39(4):527-535.

[4]. Das M, Chakraborty D (2008) Evaluation of improvement of physical and mechanical properties of bamboo fibres due to alkali treatment. J Appl Polym Sci 107(1):522-527.

[5]. Naoya Y, Kazuya O, Toori F (2004) Improvement of bending strength, fracture toughness and impact strength of bamboo fi ber composites by adding micro-fi brillated cellulose as an enhancer. Bamboo J 21:35-45.

[6]. Ramaswamy HS, Ahuja BM, Krishnamoorthy S (1983) Behaviour of concrete reinforced with jute, coir and bamboo fibres. Int $\mathbf{J}$ Cement Compos Lightweight Concrete 5(1):3-13.

[7]. https://www.researchgate.net/publication/2 59389688_Bamboo_f ibres _their_ application_in_textiles.

[8]. Jayanetti L (2000) Bamboo in construction. In: Bamboo international symposium, Chiang Mai,pp 164-174

[9]. Anping L et al (2005) The regulation role of fermenting bamboo-shoots dietary fibre in the intes-tinal flora and constipation of mice. Science and Technology of Food Industry.

[10]. Liu L, Wang Q, Cheng L, Qian J, Yu J (2011) Modifi cation of natural bamboo fi bers for textile applications. Fibres Polym 12(1):95-103

[11]. Kilinc, F. Selcen. \&quot;A review of isolation gowns in healthcare:fabric and gown properties.\&quot; Journal of engineered fibres and fabrics 10.3 (2015): 155892501501000313. 